Proceedings of the 2011 Winter Simulation Conference

S. Jain, R.R. Creasey, J. Himmelspach, K.P. White, and M. Fu, eds.

\title{
A SIMULATION TOOL TO SUPPORT RECOVERY BED PLANNING FOR SURGICAL PATIENTS
}

\author{
Yariv N. Marmor \\ Thomas R. Rohleder \\ Todd Huschka \\ David Cook \\ Jeffrey Thompson \\ Mayo Clinic \\ 200 First Street SW \\ Rochester, MN 55905, USA
}

\begin{abstract}
The cardiovascular (CV) surgery department at Mayo Clinic is planning recovery bed (ICU and step down) needs for the next 10 years. While the current practice focuses on high service level (60\% ICU utilization level), e.g., zero surgery cancelation, no shared rooms and no early discharges due to overloading; expected increasing patient volumes will require high level (system) planning in order to maintain the same level of care. A simulation model was developed to support quantitative decision making for the planning process. The model accounts for variability due to surgery scheduling (with seasonal effects), patient mix (with different growth rates), and patient length of stay (both in the ICU and the step-down unit). The model provides decision makers with a means to understand the relationship between patient service level and bed capacity/utilization level. It also provides a tool to evaluate the effects of proposed clinical and process improvements.
\end{abstract}

\section{INTRODUCTION}

Health care costs have risen dramatically in recent years. From 1980 to 2008, US per capita health care costs have grown by $700 \%$ (OECD 2010). Pressures to hold down costs is growing, with the passing of health care reform legislation clear evidence that the current financial burden of health care to society will not be allowed to grow unchecked.

The need to slow the growth or even reduce health care costs is putting significant financial pressure on health care organizations. This is particularly important to surgical practices that entail significant hospital stays, because of the high cost of both the surgery itself and the hospital services. At Mayo Clinic, institutional leadership addressed this issue in 2008 by starting an initiative to reduce per case costs by $20 \%$. Part of this effort was motivated by the recognition that for the most common cardiac surgical procedures the gap between the cost and medicare reimbursement rate was about $20 \%$. Other procedures had similar financial challenges. Cost reduction efforts originally focused primarily on operating room efficiencies, however, that focus eventually shifted to reducing the costs due to post surgery hospital stays.

One way to reduce the costs of hospital stays is to reduce the number of resources, beds, equipment, and staff associated with care for patients. To this end, the simulation model discussed in the paper was used as a planning tool to determine the number of recovery beds for Intensive Care Units (ICU) and stepdown units, called Progressive Care Unit (PCU) at Mayo Clinic. Determining the number of beds was of particular interest because significant growth for certain types of surgeries were forecast for the future. The practice wanted to know the recovery bed needs going out to 2020 considering the growth projec- 
tions. Our model allows decision makers to understand the tradeoff between the number of beds available (the "cost") and the service level to patients, defined as the availability of a cardiovascular ICU or PCU bed when needed for an incoming CV patient. Note that poor service may also have costs involved due to the lack of the prescribed level of care for patients.

Other researchers have studied the issue of determining the appropriate number of hospital beds. For example, Bekker and de Bruin (2010) discuss an Erlang loss queuing model that predicts the patient service level in hospitals depending on the number of inpatient beds. We chose to use simulation due to our desire to capture many of the details of how patients flowed from one resource to another in the CV surgery process. For example the transfer of patients from the ICU to PCU was not continuous because of the preference to avoid moving patients in the middle of the night. Further, our detailed simulation model allowed us to evaluate a variety of proposed system changes. These included, changing case volumes and mix of procedures over time, reduced lengths of stay for certain categories of patients, and the change of surgical schedules with an effort to reduce variability and its effects on downstream resource requirements. The following sections of the paper will provide a Model Description, Analysis of Results, and Conclusions.

\section{MODEL DESCRIPTION}

The Cardiac surgery division at Mayo Clinic-Rochester consists of 8 operation rooms, 33 ICU beds and 51 PCU beds on two floors. The department accommodated about 2,600 surgeries in 2010 that varied from Coronary Artery Bypass Graft surgeries (CABG) to implanting Left or Right Ventricular Assist Devices $(\mathrm{L} / \mathrm{RVAD})$ and organ transplants.

\subsection{Patient Flows}

There are three main treatment areas in the CV surgery department that we considered in our model (see Figure 1): (1) CV OR - consist of 8 operating rooms; (2) Intensive Care Units (ICU) - with 33 beds for initial recovery and stabilization after surgery; and (3) Step-down-units (PCU) which consist of 51 beds. Although most CV surgeries will result in two recovery steps - move to ICU and finish with PCU (91\%), some patients will need a complementary surgery or stabilization. A small portion of the patients require intensive care before entering surgery. The CV OR also accommodates previously transplanted patients, even if their surgery is not related to their transplant organ $(2.3 \%)$. These patients will recover outside of the CV department. In order to account for theses patients, we extend each step on Figure 1 with blue rectangles.

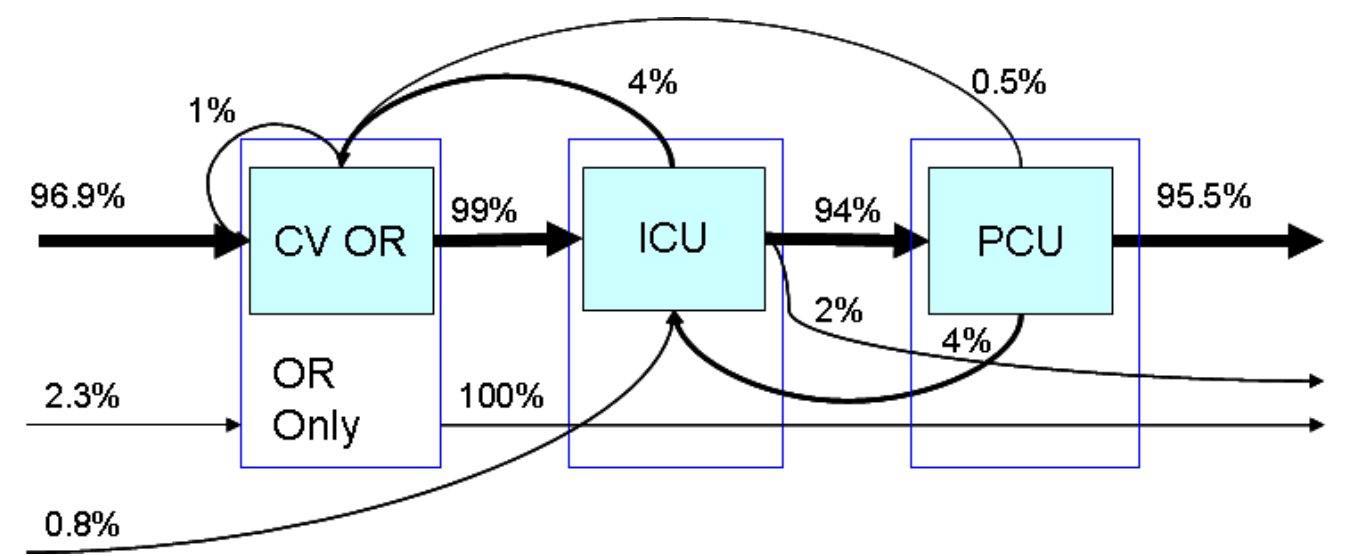

Figure 1: Patients flow in the Cardiovascular Surgery Division

There are no (practical) constraints on the daily number of surgeries in the CV OR departments, except those dictated by patients demands and surgeons preferences - the CV is a high priority department 
in Mayo Clinic, therefore additional staff or operating rooms will be provided when needed. Historical data showed that patient demand is higher in the summer and lower in the winter. We also found that on average the surgeons handled between 4 and 16 patients per day on each of the weekdays, and performed only urgent surgeries on the weekends. For baseline 2010 analysis we assume an average of 10 patients per day and used a surgical variability range from plus or minus 6 surgical procedures $( \pm 6)$ for the current surgical procedure scheduling and a low surgical variability of $( \pm 2)$ for balanced surgical procedure scheduling. For variability with the growth in surgical volume we adjust the average of surgeries, without changing the variability range.

In general, surgery duration is generally 4-6 hours. Patient length of stay (LOS) in the ICU is shorter (approximately 2 days) than the time in the PCU (approximately 4-5 days). Acute patients or those with medical complications can experience much longer stays in both the ICU and PCU. More careful analysis of patient LOS in the recovery units revealed that this time is a combination of three components: (1) the time required for CV clinical care (unique to CV surgeries); (2) Recovery care (which is not unique to $\mathrm{CV}$, but to most ICU or PCU patients); and (3) time due to process policies and preferences - for example, patients are not transferred from ICU to PCU after midnight, and patients waiting for discharge from the PCU to certain assisted care facilities on Saturdays and Sundays cannot be transferred because new patients are not accepted on weekends.

The number of recovery beds is found to be a potential bottleneck for the CV department. Although patient diversion is an option in case of an overload, the $\mathrm{CV}$ department wishes to avoid this as much as possible in order to deliver a high level of clinical and nursing service.

\subsection{Data Sources and Description}

Because not all types of surgical procedures result in similar lengths of stay the patients were categorized into seven groups; Transplant, VAD (Ventricle Assist Device), ECMO (Extracorporeal Membrane Oxygenation), Pediatric, Robotic Assist, Rapid Recovery, and Other Adult. Pediatric patients were classified as all patients less than 18 years old, and Rapid Recovery patients were identified separately by CV staff prior to surgery as those that can recover more quickly than others.

Approximately 1,500 surgical cases with known classification types were available. However, this was found to be insufficient for generating reliable probability and LOS distributions for rare surgical procedures, such as transplants. Therefore, approximately 15,000 ICU admissions from 2005 through March of 2011 were used, coupled with a Decision Support Services (DSS) data, which held patient surgical procedure information (ICD9), to augment patient classification groups. SAS (SAS Institute 1999) was used to merge the two databases in order to find these patient classifications.

Statistical analysis was also performed using SAS to examine the average LOS for each patient type. These results were verified by experts within the ICU and PCU, and also with experts who are familiar with surgical procedure coding (ICD9) to ensure that our classifications were correct. ExpertFit (Law and McComas 1999) was then used to generate patients LOS distributions in the ICU and PCU for each classification group.

\subsection{Model Validation}

We validated our model by comparing the empirical ICU and PCU utilization levels with the simulation average, lower- and upper- bound (see Figure 2). Note that the ICU load increases during the weekdays while scheduled surgeries are performed and then drops over the weekends. The difference between Sunday and Friday ICU utilization level is more than $50 \%$. The load in the PCU is more evenly spread because of the longer LOS, but we can still see that it lags the ICU load by about one to two days (ICU average LOS).

Additionally, we also validated the ICU and PCU bed distribution, and found that the best fit at the target service level (or Quality of Service, QoS) would be to determine the number of beds such that 95 
percent of the time the ICU and the PCU could accommodate new patients without the need to divert them to an alternative location.

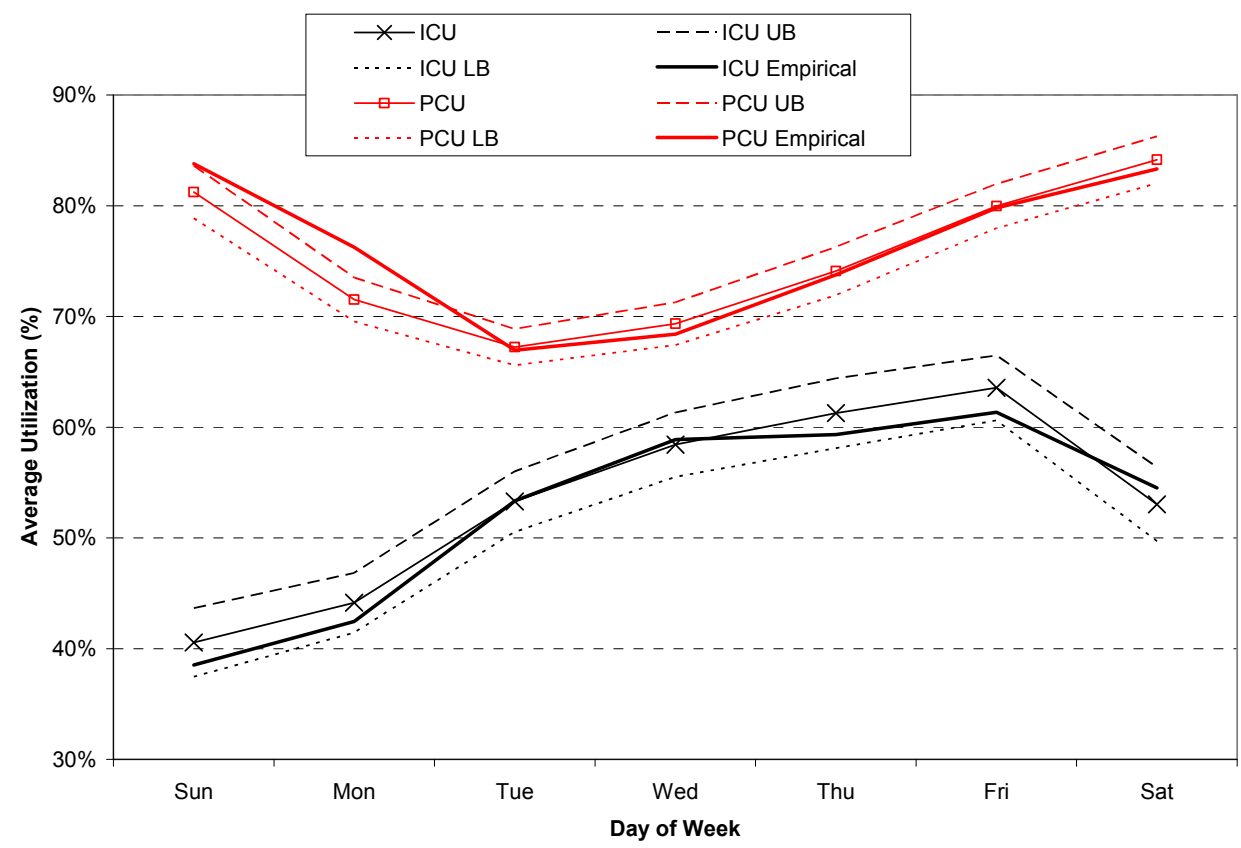

Figure 2: Validation of ICU and PCU simulation and empirical utilization level

\section{RESULTS}

\subsection{Experimental Design}

The model was run over a 10 year horizon: 2010 to 2020. In order to account for rare events and extended Length of Stay (LOS), we ran a warm up period of 3 months for each replication and then we collected data for a whole year. We then ran 10 replications for each scenario for each year for a total of 110 replications per scenario. We collected the number of beds needed for the target Quality of Service (QoS = $0.95)$ operating with current surgery variability levels at $( \pm 6)$ and $( \pm 2)$, and the starting average of approximately 10 surgeries per day (in 2010). We also ran replications with and without scheduling a Saturday morning shift of surgeries for a total of 880 replications.

The results of the average number of beds saved by each scenario over all the projection years compared with the current practice (variability \pm 6 , no Saturdays surgeries) are presented in Table 1 . The number of beds needed for ICU and PCU by year are presented in Figure 3 and Figure 4 respectively.

Table 1: Average Number of ICU and PCU Beds Saved Compared to Current Practice

\begin{tabular}{|l|r|r|}
\hline & ICU & PCU \\
\hline variability $( \pm 6)$, with Saturday surg. & 1.5 & 1.4 \\
\hline variability $( \pm 2)$, without Saturday surg. & 1.1 & 1.7 \\
\hline variability $( \pm 2)$, with Saturday surg. & 2.5 & 2.6 \\
\hline
\end{tabular}




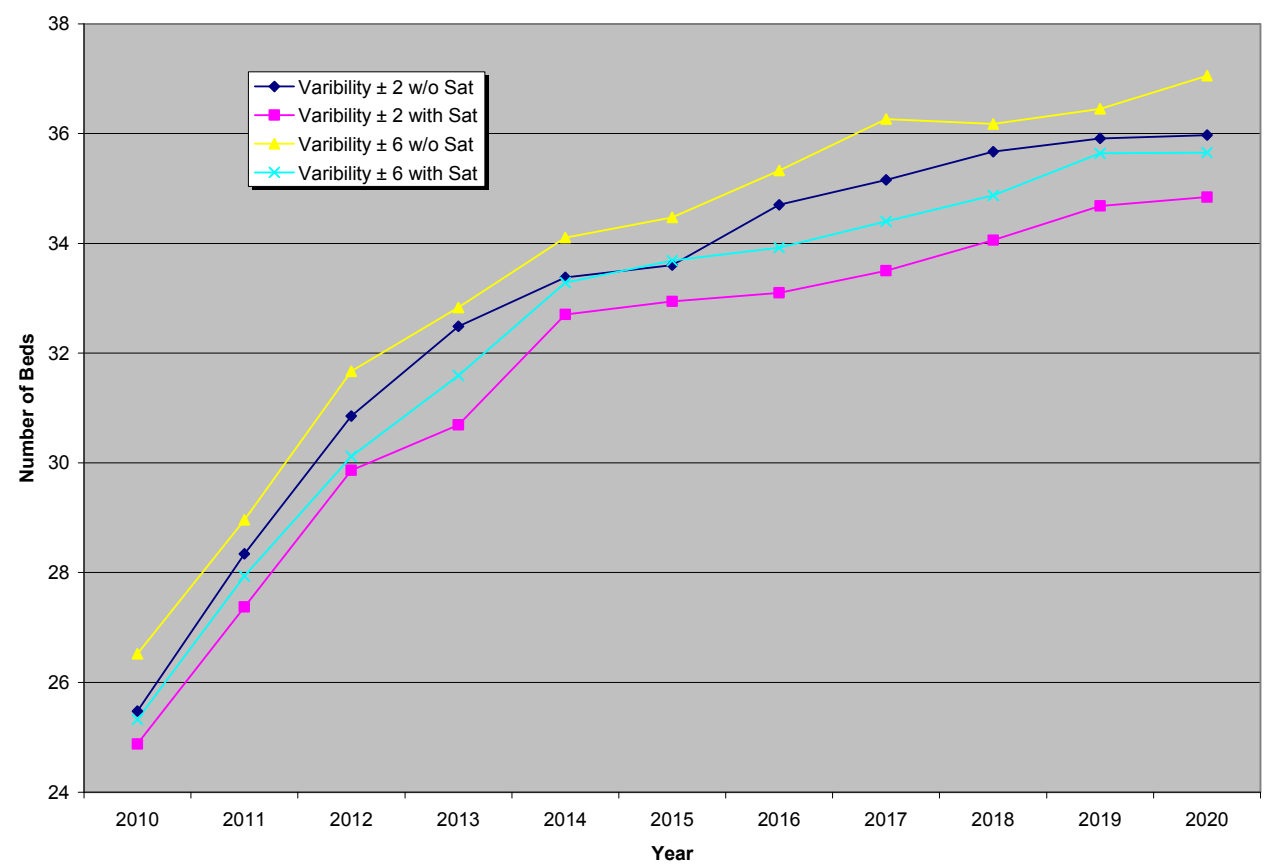

Figure 3: Average of ICU beds needed for each scenario by year

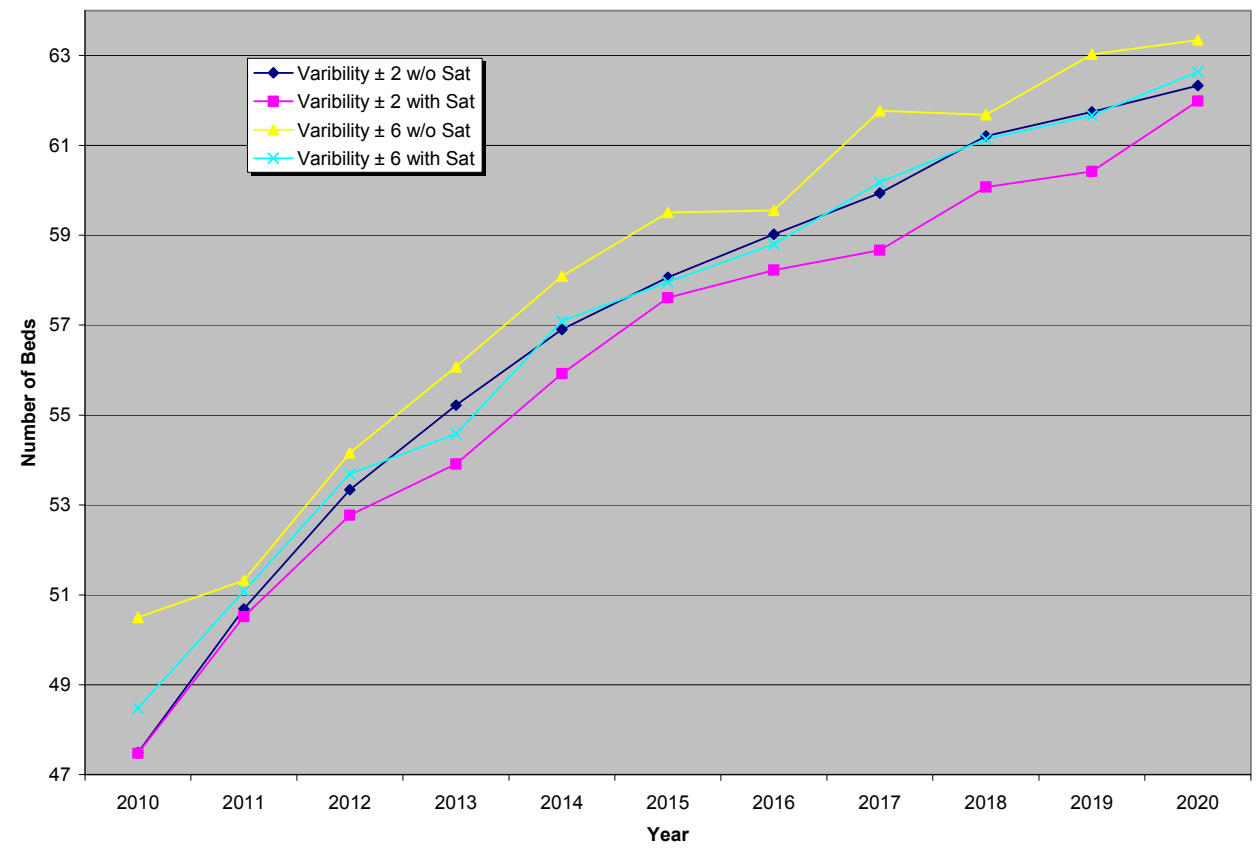

Figure 4: Average of PCU beds needed for each scenario by year

\subsection{Effects of Reducing Weekday Surgery Variability}

We started by looking on the variability effect on the number of beds needed in the ICU and in the PCU without incorporating Saturday surgeries. Table 1 shows that an average of 1.5 and 1.4 beds from the ICU 
and PCU, respectively can be saved ( $\mathrm{p}$-value $<0.001$ ); however, reducing variability did not save any beds in $24 \%$ of the replications.

\subsection{Adding Saturday Surgeries}

We further analyzed the impact of Saturday surgeries on case variability. As we see in Table 1, the impact of performing surgeries on Saturday mornings enhanced the effect of surgical case variability reduction (p-value $<0.001$ ).

Because both weekday surgery variability and Saturday surgery parameters influence the overall average daily surgery variability, it is useful to see how each impacts overall variability of beds needs. For that, we calculated the standard deviation of the four options ( \pm 2 and \pm 6 variability, and with and without Saturday surgeries). From Table 2 we see that the lowest overall daily variability happens when we use weekdays variability of $( \pm 2)$ and perform Saturday surgeries. This option yields the lowest SD and the greatest bed reduction. Although we see that Saturday surgeries $( \pm 6)$ and Without Saturday surgeries $( \pm 2)$ (Table 1) have an opposite effect on ICU and PCU bed gains, the SD of the two options are very close $(4.689,4.622$ respectively) and the two options are not statistically significantly different.

Table 2: Standard Deviation (SD) for the Overall Daily Number of Surgeries

\begin{tabular}{|l|c|c|}
\hline Weekday variability & with Saturdays & without Saturdays \\
\hline average $( \pm 6)$ & 4.689 & 5.386 \\
\hline average $( \pm 2)$ & 3.459 & 4.622 \\
\hline
\end{tabular}

\section{CONCLUSIONS}

Recovery beds for surgical patients are an expensive healthcare resource. Average costs for ICU patients can be nearly $\$ 4,000$ per day (Dasta et al. 2005). Therefore, ensuring the correct number of beds are available and staffed is an important decision for hospital administrators. In this paper we report on a simulation model used at Mayo Clinic to help decision makers determine the number of recovery beds needed for cardiovascular surgery patients. The model was able to explicitly show the relationship between quality of service (availability of beds to incoming patients) and the number of beds included in ICU and PCU (step down unit).

Scenarios that reduced the variability in daily scheduled surgeries were also evaluated. The model showed that about 5 total recovery beds can be saved by reducing weekday surgical case variability from \pm 6 to \pm 2 and performing Saturday morning surgeries. The model also reported that adding the Saturday surgeries reduced case variability to about the same level as reducing weekday surgery variability by a factor of three.

Future work will consider changing the patient mix over the week to minimize bed needs subject to an appropriate service level constraint. The model will also be used to explore the effects of reduction in PCU length of stay as well as transfers of patients from the CV ICU who no longer required CV related critical care.

\section{REFERENCES}

Bekker, R., and A. M. de Bruin. 2010. "Time-Dependent Analysis for Refused Admissions in Clinical Wards." Annals of Operations Research 178:45-65.

Dasta, J. F., T. P. McLaughlin, S. H. Mody, and C. T. Piech. 2005. "Daily Cost of an Intensive Care Unit Day: The Contribution of Mechanical Ventilation." Critical Care Medicine 33: 1266-1271.

OECD (Organization for Economic Cooperation and Development). 2010. Health Data 2010 Report.

Law, A. M., and M. G. McComas. 1999. "ExpertFit: Total Support for Simulation Input Modeling." In Proceedings of the 1999 Winter Simulation Conference, edited by P. A. Farrington, H. B. Nembhard, 
D. S. Sturrock, and G. W. Evans, 261-266. Piscataway, New Jersey: Institute of Electrical and Electronics Engineers, Inc.

SAS Institute. 1999. SAS/STAT User's Guide, Version 8. SAS Institute. Cary, North Carolina, USA.

\section{AUTHOR BIOGRAPHIES}

YARIV N. MARMOR is a Postdoctoral Fellow in the Division of Health Care Policy and Research at Mayo Clinic. He holds a PhD in Industrial Engineering from the Technion-Israel Institute of Technology. His current research interests are in the area of process analysis and improvement of medical systems. Dr. Marmor has published his research in journals such as ACM Transactions on Modeling and Computer Simulation (TOMACS), International Journal of Production Research, IIE Transactions, and Journal of Health Organization and Management. His email address is marmor.yariv@mayo.edu.

THOMAS R. ROHLEDER is a Professor of Health Care Systems Engineering in the Division of Health Care Policy and Research at Mayo Clinic. He was as previously a professor of Operations Management in the Haskayne School of Business at the University of Calgary. He has a Ph.D. in Business Administration, and B.S.B. in Finance from the University of Minnesota. Before obtaining his doctorate, Dr. Rohleder worked in the financial services sector for ITT Commercial Finance Corp., and Northwestern National Life Insurance Co. Dr. Rohleder has over 40 publications in journals such as the Journal of Operations Management, Production and Operations Management, and Health Care Management Science. He is currently working on research in the areas of health systems engineering and health care operations management. His email address is rohleder@mayo.edu.

TODD R. HUSCHKA is a Master Health Systems Analyst in the Division of Health Care Policy and Research at Mayo Clinic. He has been at the Mayo Clinic for 15 years, with the past 6 years focusing on Operations Research. He has a MS in Industrial Engineering from the University of Wisconsin, and a BS in Statistics from North Dakota State University. His primary focus of research has been in the simulation/optimization of surgical and clinical schedules as well as the optimization of hospital resources. He has several publications and has been regular presenter at both INFORMS and WSC. His email is huschka.todd@mayo.edu.

DAVID J. COOK, MD is a Professor of Anesthesiology, Mayo Medical School and is the Chair of the Division of Cardiovascular Anesthesia. He received his Doctor of Medicine from Jefferson Medical College in Philadelphia, and completed his internship, residency, research training (Cardiovascular Pharmacology) and Cardiovascular Anesthesiology Fellowship at the Mayo Graduate School of Medicine in Rochester, MN. Dr. Cook has authored/co-authored over 80 peer-reviewed publications and book chapters and has edited two books (Innovations in Cardiovascular Care - DVD Monograph - 2006 and Cardiopulmonary Bypass, Cambridge University Press, Ed. Ghosh, Falter \& Cook - 2009). He is an expert in cerebral blood flow/metabolism and cardiac surgical brain injury and speaks nationally and internationally on anesthesia for cardiovascular surgery. Over the last three years Dr. Cook has helped lead practice redesign in cardiovascular surgery at Mayo-Rochester. His email address is cook.david@mayo.edu.

JEFFREY E. THOMPSON is a Senior Health Systems Engineering Analyst in the Division of Systems and Procedures at Mayo Clinic. Jeff's work has primarily focused on projects in Cardiovascular Surgery Redesign and Handoff Communication. Jeff also serves as a faculty instructor for Mayo Clinic's Quality Academy teaching various quality improvement courses. Prior to joining Mayo, he worked as a Six Sigma Black Belt within the Lean Six Sigma Department at the Memorial Hermann Healthcare System in Houston, Texas. Jeff also worked as a physical therapist with clinical inpatient rehabilitation experience. He received his MHA from Texas Woman's University in Houston, Texas and his MPT from Ohio University in Athens, Ohio. Jeff's email address is thompson.jeffrey2@mayo.edu. 